\title{
Dictynna
}

Dictynna

Revue de poétique latine

15 | 2018

Varia

\section{Otium e letteratura nei Carmina Priapea (e in} Marziale)

\section{Alfredo Mario Morelli}

\section{(2) OpenEdition}

\author{
Journals
}

Edizione digitale

URL: http://journals.openedition.org/dictynna/1525

DOI: 10.4000/dictynna.1525

ISSN: 1765-3142

Notizia bibliografica digitale

Alfredo Mario Morelli, « Otium e letteratura nei Carmina Priapea (e in Marziale) », Dictynna [En ligne], 15 | 2018, mis en ligne le 21 décembre 2018, consulté le 10 décembre 2020. URL : http:// journals.openedition.org/dictynna/1525; DOI : https://doi.org/10.4000/dictynna.1525

Questo documento è stato generato automaticamente il 10 décembre 2020.

\section{(c) (i) (9)}

Les contenus des la revue Dictynna sont mis à disposition selon les termes de la Licence Creative Commons Attribution - Pas d'Utilisation Commerciale - Pas de Modification 4.0 International. 


\title{
Otium e letteratura nei Carmina Priapea (e in Marziale) ${ }^{1}$
}

\author{
Alfredo Mario Morelli
}

\section{Il poeta otiosus dei Carmina Priapea : un problema di inter- e intratestualità.}

1 Nel secondo epigramma dei Carmina Priapea, presentando i suoi versi al dio, l'ego afferma di averli composti otiosus (v. 9), insomma in una esibita condizione di otium che va vista sullo sfondo di una lunga tradizione letteraria, soprattutto (anche se non solo) d'ambito epigrammatico. Quello che impressiona è soprattutto la costellazione di motivi in cui tale elemento si colloca : nel presentare la raccolta al lettore (Priap. 1) e nell'offrirla a Priapo (Priap. 2), l'ego si presenta non solo come poco laboriosus $(2,3) \mathrm{ma}$ anche come autore di carme incomptum (questo tratto è addirittura posto in incipit, 1,1: carminis incompti). L'intero dispositivo proemiale mostra grandi analogie con i due epigrammi incipitari di Stratone, ed esse sono state oggetto di importanti studi anche recenti ${ }^{2}$ : in questo articolo, intendo dimostrare che sono proprio le rimarchevoli differenze di contestualizzazione del motivo dell'otium (unito non solo a quello dello scarso labor, ma soprattutto a quello della 'rozzezza' del canto, in omaggio alla rustica divinità priapica, ben diversa da quelle cui si rivolge Stratone) che rendono abbastanza chiara una volontà allusiva (per 'straniamento') da parte del poeta dei Priapea nei confronti dell'autore greco ( $\$ 2$ infra). Soprattutto, però, nei due carmi iniziali della raccolta latina è evidente (a mio parere) il debito nei confronti di Marziale : il tema dell' otium si offre, all'interno dell'epigrammatica marzialiana, in un variegato ventaglio di motivi che dovranno essere attentamente considerati per cogliere il senso profondo della rielaborazione letteraria da parte del poeta dei Priapea ( $\$ 3$ infra). Una volta effettuato questo percorso, io credo apparirà chiaro quanto le risonanze del motivo dell'otium, nelle sue sinergie con gli altri che fin dall'inizio della raccolta sono stati posti in relazione con esso (lusus, assenza di labor, rusticitas, anti-estetica del libellus), caratterizzino l'intera raccolta priapica, in un continuo dialogo (spesso nella forma, 
ancora, di una allusività 'straniante') con gli auctores dell'epigramma latino (Catullo e Marziale innanzitutto) : tutto ciò contribuisce a rafforzare l'idea che l'autore dei Priapea sia unico e che abbia operato in età successiva all'epoca di composizione dei libri di Marziale (§). Ma procediamo con ordine.

\section{Il doppio proemio dei Priapea}

\section{Priap. 1}

Carminis incompti lusus lecture procaces, conveniens Latio pone supercilium.

non soror hoc habitat Phoebi, non Vesta sacello, nec quae de patrio vertice nata dea est,

sed ruber hortorum custos, membrosior aequo, qui tectum nullis vestibus inguen habet.

aut igitur tunicam parti praetende tegendae, aut quibus hanc oculis aspicis, ista lege.

$\mathrm{Tu}$ che stai per leggere battute salaci di un rozzo carme, non alzare il sopracciglio, che s'addice al Lazio.

In questo sacello non abita la sorella di Febo, o Vesta, né la dea nata dalla testa del padre,

ma un rubizzo custode dell'orto, più dotato del normale, che ha l'inguine non coperto da veste.

E allora, o metti una tunica sulla parte da coprire, o leggi questi versi con gli stessi occhi con cui osservi quella.

\section{Priap. 2}

Ludens haec ego teste te, Priape, horto carmina digna, non libello, scripsi non nimium laboriose.

nec Musas tamen, ut solent poetae, ad non virgineum locum vocavi : nam sensus mihi corque defuisset, castas, Pierium chorum, sorores auso ducere mentulam ad Priapi. ergo quidquid id est, quod otiosus templi parietibus tui notavi,

in partem accipias bonam, rogamus. ${ }^{3}$ Scherzando (sei testimone), o Priapo, ho scritto questi versi degni d'un giardino, non di un libro, senza troppo impegno. Non ho chiamato le Muse, come fanno i poeti, in un luogo poco virginale: mi sarebbero mancati animo e cuore se avessi osato condurre le caste sorelle, coro danzante di Pieria, al fallo di Priapo. E perciò, qualunque sia il valore di ciò che ozioso ho scritto sui muri del tuo tempio, chiedo che tu l'accolga con benevolenza.

2 Nel suo studio sull'argomento ${ }^{4}$, Mario Citroni ha messo giustamente in evidenza come i carmi 1-2 vadano considerati un dittico proemiale in sé perfettamente integrato e ripreso poi, 'al mezzo', dal c. 49 : i due componimenti iniziali non trovano la loro ratio in una opposizione tra apostrofe al lettore (c. 1) e funzione dedicatoria (c. 2), come voleva Vinzenz Buchheit (l'opposizione esiste, ma non esaurisce certo le motivazioni metapoetiche di questo sofisticato dispositivo iniziale $)^{5}$; piuttosto, essa va cercata 
nell'integrazione di due momenti apologetici, l'uno destinato a dar ragione dell'oscenità verbale, l'altro della mancanza di elaborazione formale. Citroni propone un'analisi molto efficace delle corrispondenze tra Priap. 1 e tutta la sequenza iniziale del I libro di Marziale, fino al c. 4 (cf. soprattutto Priap. 1,2 conueniens Latio pone supercilium e Mart. 1,4,2 terrarum dominum pone supercilium ${ }^{6}$, 'abbassa il sopracciglio signore del mondo', ove giustamente lo studioso conclude per la seriorità del testo dei Priapea rispetto a quello marzialiano ${ }^{7}$ ), sia tra Priap. 2 e Catullo 1 , ove l'omogeneità di struttura disvela le differenze di poetica tra i due testi ${ }^{8}$. Citroni mette giustamente in evidenza il significato decisivo di questi ipotesti, quando l'autore dei Priapea, in sede proemiale, mette a fuoco la questione della poetica; egli sottolinea le indubbie differenze che distanziano i due carmi priapici dal dittico iniziale, che pure è stato spesso portato a confronto, della Paidikè Moûsa di Stratone (AP 12,1-2) $)^{9}$. Sono d'accordo con questa analisi, ma d'altro canto forse proprio le notevoli somiglianze e, esattamente, le differenze tra la coppia proemiale della raccolta priapica e quella stratoniana (in buona parte rilevate da Buchheit ${ }^{10}$ ) aiutano a comprendere le caratteristiche particolari dell'operazione proposta dall'anonimo autore latino.

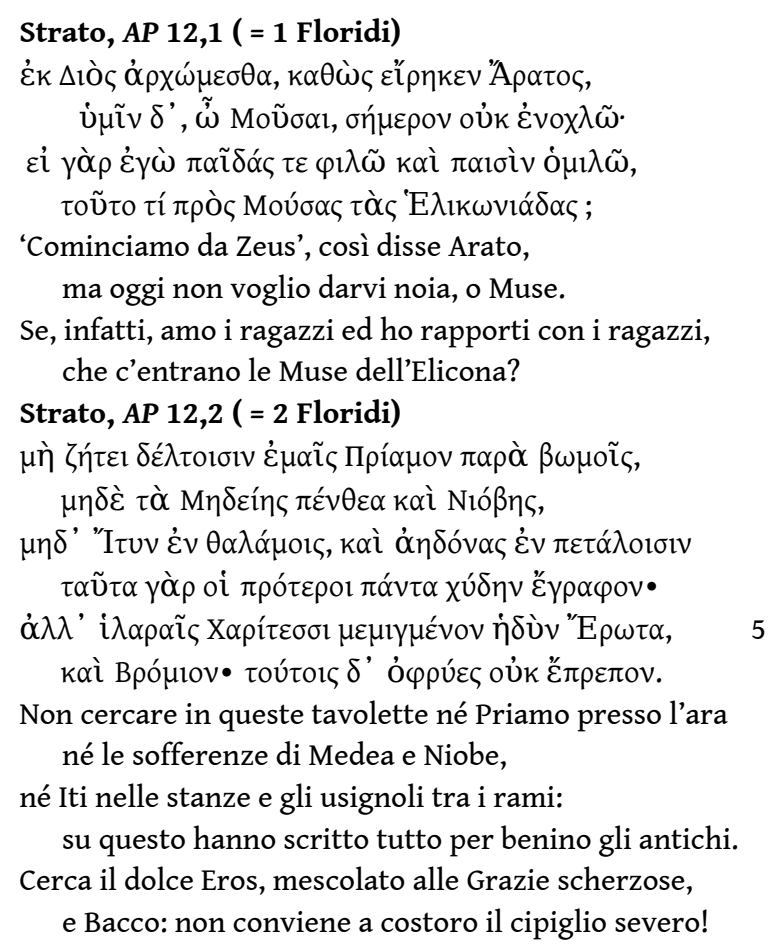

e Bacco: non conviene a costoro il cipiglio severol

Se richiamiamo lo schema incipitario apostrofe al lettore + invocazione al dio, ci accorgiamo che Stratone organizza il suo dittico in sequenza inversa rispetto a quello dei Priapea : a una dedica, ancorché scherzosa, al dio, cioè Zeus (l'incipit arateo di Anth. Gr. 12,1, nella sua parodica solennità, è da considerare una dedica a tutti gli effetti, in una forma ellittica che va 'integrata' con la memoria letteraria del testo di Arato), segue una apostrofe al lettore, che è invitato a non cercare nella poesia stratoniana figure del mito (e della poesia elevata) incongrue rispetto alla levità giocosa dell'eros paidico (analogo motivo in Priap. 1 : la struttura di Anth. Gr. 12,2,1-5, $\mu \grave{\eta} \ldots \mu \eta \delta \grave{\varepsilon} \ldots \mu \eta \delta^{\prime}$... $\alpha \lambda \lambda \lambda^{\prime}$, mostra indubbie somiglianze con Priap. 1,3-5 non ... non ... nec ... sed ..., sia pure con le differenze retoriche del caso). L'invito al lettore da parte di Stratone in AP 12,2,6

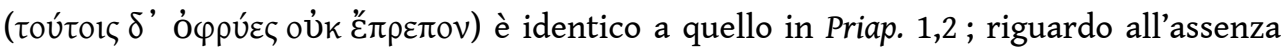
delle Muse, il pretesto (almeno il pretesto) accampato in tutti e due i casi è che le dee 
sono estranee all'argomento : in Stratone, ad esse non conviene la poesia paidica (AP 12,1,2-4 e, implicitamente, anche 12,2,5, con la menzione delle Cariti in luogo delle Muse, per quanto le Grazie abbiano anch'esse una loro dignità di divinità ispiratrici), mentre nei Priapea, ad esse, in quanto virgines, non si confà la mentula priapica (Priap. 2,4-8). Tanto più, in queste analogie, si esaltano le differenze di strategia apologetica tra Stratone e i Priapea, ben rilevate da Citroni proprio a partire dalla diversa funzione della mancata "invocazione alle Muse ${ }^{11}$. Nella retorica incipitaria stratoniana, al contrario che in quella del Priapo latino, non si crea una costellazione tra i due motivi del lusus letterario e della rozzezza espressiva (rusticitas) ed emblema di tutto questo è che Stratone può invocare in suo soccorso figure tutt'altro che secondarie del pantheon greco (a parte Zeus e le Cariti, la sua è poesia di Eros e di Bromio, cf. AP 12, 2, 5-6) ${ }^{12}$, mentre l'autore latino, orfano delle Muse, pone sotto il segno di Priapo i suoi versi incompti. La dialettica che si apre tra il carattere 'esile', giocoso dei versi priapici e la loro dichiarata rozzezza 'rustica' crea poi nel corso della raccolta effetti umoristici, nel confronto spesso adombrato con l'estetica del carme breve e sofisticato d'impronta callimachea ${ }^{13}$. Se si vanno poi a considerare le altre attestazioni antiche del motivo incipitario del 'congedo delle Muse' (con allocuzione alle medesime o meno), si coglie appieno la particolarità del proemio dei Priapea. Stazio, nel genere 'minore' delle Silvae, vi fa spesso ricorso, ma certamente non è mai in questione una poetica della rusticitas, della rozzezza incondita, quanto piuttosto dell'adeguatezza dell' 'ispirazione' (sempre da vates professionista) al tema della poesia ${ }^{14}$; è facile vedere come l'autore di Priap. 1-2 porti alle estreme conseguenze esattamente questo elemento. A un dio rusticus come Priapo non si possono che dedicare versi rozzi ; e l'assenza delle Muse si giustifica, certo, con motivi di convenienza, a fronte di contenuti di rara oscenità (la loro estraneità alla sconcia materia del canto è, in effetti, apparentemente come in Strato $A P$ 12,1,3-4, l'unica causa addotta in Priap. 2,4-8), ma comporta anche, coerentemente, che la poesia che si coltiva nell'hortus Priapi sia rozza e incondita. Il gioco delle (notevoli) analogie e delle (altrettanto notevoli) differenze rende a mio parere probabile l'ipotesi che qui un intento allusivo al doppio proemio stratoniano ci sia: certo, in forma 'straniante', ammiccando al lettore colto. Naturalmente è ipotesi da avanzare con tutte le cautele del caso.

4 Un ulteriore elemento che va preso in considerazione in questo articolato sistema proemiale è il ruolo che il poeta assegna all'otium rispetto alla sua stessa attività di versificatore. Alla luce di esso, meglio si comprende la evidente, quanto non banale, operazione di ripresa dei grandi modelli epigrammatici che l'autore ha inteso proporre al suo pubblico : Catullo, Marziale e, probabilmente, Stratone stesso. In Priap. 2,9-11 ergo quidquid id est, quod otiosus / templi parietibus tui notavi, / in partem accipias bonam, rogamus, la iunctura ergo quidquid id est allude sicuramente a Catull. 1,8 quare habe tibi quidquid hoc libelli, ma l'invito al dio Priapo acquisisce un significato molto diverso rispetto a Catullo ${ }^{15}$. Il poeta ha composto 'nell'ozio', otiosus: tale connotazione va attentamente considerata alla luce della tradizione letteraria sul motivo. Già al v. 3 scripsi non nimium laboriose viene segnalata una differenza essenziale rispetto a Catull. 1, in quanto Catullo, elogiando l'opera storiografica di Cornelio Nepote, afferma che l'opera dell'amico è composta (esattamente al contrario) di chartae ... laboriosae ${ }^{16}$; il poeta dei Priapea al v. 9 precisa poi il concetto con il termine otiosus, posto, come laboriose, in chiusura di endecasillabo. L'otium è qui non solo il tempo lasciato libero dalle attività politiche o dagli affari privati, bensì il trastullo poco o per nulla 'faticoso', non impegnativo. In Catull. 1 , alla futilità dei contenuti o alla umiltà delle forme 
letterarie corrispondono eleganza, novità di forma, raffinatezza del liber-manufatto e, senza dubbio, dei versi in esso compresi (cfr. soprattutto vv. 1-2 lepidum, novum libellum / arida modo pumice expolitum ; cfr. poi 4 meas esse aliquid putare nugas) ; sia pure solo di riflesso, s'intravede anche un ideale di 'fatica' (v. 7 laboriosae) : il ruolo, il prestigio del lettore-Nepote, il suo stesso labor nell'attività letteraria divengono garanzia della qualità delle nugae del poeta, destinate a sfidare i saecla (Catull. 1,9-10). Nei Priapea all'esilità fa riscontro non solo la rozzezza dei versi, ma anche l'ozio' inteso come mancanza di impegno e di labor, neppure riscattata e 'sublimata' da un lettore illustre e 'laborioso', che dia il suo avallo. Insomma, nel nuovo dispositivo metapoetico viene completamente disarticolato quel paradosso del labor in tenui, dell'impegno nel piccolo, nell'esile' che costituisce l'essenza della proposta neoterica ${ }^{17}$. Ne deriva una ridefinizione dei rapporti tra poeta e lettore, tra letteratura, libro, dottrina filologica e otium.

\section{L'otium poetico nella poesia marzialiana.}

L'otium così definito non ha nulla a che fare non solo con il concetto di 'disimpegno operoso' sotteso alla poetica neoterica, ma anche con altre formulazioni cui pervennero in epoca successiva i poeti di età augustea o del primo secolo dell'Impero ${ }^{18}$. Come è noto, in Virgilio e Orazio, l'otium è uno spazio conquistato dalla saggezza del poeta e garantito dal potere del principe : è lo spazio in cui Titiro può intonare la sua musa in onore di Amarillide (Verg. Ecl. 1,6 o Meliboee, deus nobis haec otia fecit, 'o Melibeo, un dio ci ha creato questi ozi'), oppure il piccolo mondo in cui il poeta può condurre la sua vita sobria, dedita alle Muse e lontano dal volgo (Hor. Carm. 2,16,1 ss. otium divos rogat in patenti / prensus Aegaeo ... mihi parva rura et / spiritum Graiae tenuem Camenae / Parca non mendax dedit et malignum / spernere volgus, 'pace è quel che chiede agli dei chi è sorpreso nel mezzo dell'Egeo ... a me la Parca non menzognera ha dato un piccolo campo e lo spirito sottile di una greca Camena, e il disprezzo per il volgo malvagio'; cfr. anche Sat. 1,7,36 otia ... liberrima, 'gli ozi ... più che liberi'; Ars 199 etc.) ${ }^{19}$. Per arrivare ad epoche, a generi letterari e ad opere che più direttamente influenzano i Priapea, Marziale esprime spesso il lamento della nuova condizione del poeta-cliente che, tutto preso dai suoi obblighi sociali con i patroni e con le necessità del vivere quotidiano nell'Urbe, non ha tempo e modo di comporre poesia ${ }^{20}$. Nel primo libro $(1,107)$ Marziale si 'giustifica' per la sua inerzia (desidia), reclamando per sé gli otia di cui godettero proprio Virgilio e Orazio, grazie a Mecenate ${ }^{21}$ :

Saepe mihi dicis, Luci carissime Iuli,

'Scribe aliquid magnum : desidiosus homo es.'

Otia da nobis, sed qualia fecerat olim

Maecenas Flacco Vergilioque suo :

Condere victuras temptem per saecula curas $\quad 5$

Et nomen flammis eripuisse meum.

In steriles nolunt campos iuga ferre iuvenci :

Pingue solum lassat, sed iuvat ipse labor.

Spesso mi dici, carissimo Lucio Giulio,

'Scrivi qualcosa di grande: sei un fannullone.'

Donami gli ozi, ma quelli che un tempo procurò

Mecenate a Flacco e al suo Virgilio:

proverei a comporre un'opera destinata a sfidare i

secoli e a strappare il mio nome al rogo. 
I giovenchi non vogliono trascinare l'aratro in

campi sterili; la terra fertile affatica, ma è il lavoro stesso che dà piacere haec otia fecit (cfr. anche subito dopo, qualia fecerat); ma non è lo spazio di un genere 'umile' come quello bucolico che Marziale intende aprire. Egli gioca in modo sottile, grazie all'enfatico accostamento ai vv. 2-3 desidiosus homo / otia, sul significato 'deteriore' di otium ${ }^{22}$ : desidera che esso gli venga concesso, ma non chiede certo quello desidiosum. Come si vede, gli otia qui favorirebbero, in realtà, un'attività poetica in generi più elevati, un labor duro, ma fruttuoso, in un terreno fertile ${ }^{23}$. Se ne deduce che la mancanza di otium protetto da patroni benevoli diviene per Marziale il motivo per cui egli si dedica ad un genus non magnum né impegnativo, l'epigramma ${ }^{24}$. Il richiamo implicito alle dure condizioni di vita del poeta permette, da un lato, di inquadrare in modo ben diverso il classico motivo (frequente soprattutto nell'elegia ${ }^{25}$ ) dell'inertia che caratterizzerebbe i generi minori rispetto a quelli elevati, dall'altro di riproporre un'idea di otium 'impegnato', l'esatto contrario rispetto alla desidia (significativa la parola finale del carme, labor). L'asse del discorso si sposta sull'antitesi 'otium operoso del poeta protetto da un mecenate, che scrive grande poesia vs inerzia forzata (cfr. v. 7) del povero poeta-cliens che scrive epigrammi' ${ }^{26}$. Il motivo si intreccia (arricchendolo e complicandolo notevolmente) con il tema, più generale, del contrasto tra l'otium raccolto e modesto, 'oraziano', del piccolo podere e gli officia della grande Urbe, già ben presente nel I libro (cfr. ad es. 1,55) ${ }^{27}$. In 1,107 la mancanza di otium non permette di dedicarsi a generi elevati, come se la faticosa e dispersiva vita urbana del cliente concedesse la possibilità di impegnarsi solo nell'epigramma ; ma Marziale preciserà e, anzi, modificherà successivamente questo motivo.

In 8,3, quando Marziale annuncerà (in modo artificioso) il proposito di abbandonare l'epigramma, l'umile musa Talia insorgerà con queste sorprendenti parole (vv. 11-18) ${ }^{28}$ :

'Tune potes dulcis, ingrate, relinquere nugas? Dic mihi, quid melius desidiosus ages?

An iuvat ad tragicos soccum transferre coturnos, Aspera vel paribus bella tonare modis,

Praelegat ut tumidus rauca te voce magister Oderit et grandis virgo bonusque puer?

Scribant ista graves nimium nimiumque severi, Quos media miseros nocte lucerna videt.'

'Tu forse puoi lasciare, ingrato, le dolci sciocchezze? E dimmi, cosa farai di meglio, da pigro che sei?

Oppure vuoi trasformare il socco in tragici coturni e far rimbombare le aspre guerre in ritmi sempre

uguali, perché con voce roca ti legga il maestro, ridondante, e ti odino la ragazza già grande e il

bravo fanciullo? Queste cose le scrivano quelli troppo seri e troppo severi, che la lucerna vede miseri di notte'.

8 Che cosa significa esattamente desidiosus al v. 12 ? Due sono le ipotesi in campo :

1) Secondo alcuni studiosi, il motivo è esattamente rovesciato rispetto a $1,107^{29}$ : Marziale sarebbe desidiosus perché si congeda dal genere epigrammatico ; Talia si sente, in sostanza, 'abbandonata' dal suo poeta, per pigrizia ${ }^{30}$;

2) secondo altri, Marziale viene chiamato desidiosus non perché non sia più poeta epigrammatico, ma esattamente perché lo è o, comunque, perché tali sono i suoi costumi abituali: 'cosa potrai fare di meglio, da pigro quale sei ?'. Di conseguenza, 
Christian Schöffel traduce il v. 12 : «sag mir, was willst du denn lieber tun, ohne dich dabei anstrengen zu müssen? $»^{31}$.

9 Non è facile proporre argomenti davvero decisivi a favore dell'una o dell'altra interpretazione. Nel discorso che l'ego poetico fa ai vv. 1-8, egli non dichiara espressamente di voler passare a generi poetici più elevati, ma semplicemente che egli non vuol più fare epigrammi o poesia tout court : sicché potrebbe benissimo darsi che Talia risponda 'in due tempi' a questa dichiarazione dell'ego, dapprima accusandolo di 'pigrizia' non militante e 'diserzione', perché non vuole più comporre carmi, in seguito, invece, facendo l'ipotesi che egli voglia, in realtà, dedicarsi a poesia epico-tragica (vv. 12-13 quid melius ... ages? an iuvat ad tragicos etc.) ${ }^{32}$. D'altro canto, desidia e desidiosus sono termini che, più comunemente, si utilizzano in riferimento alla poetica elegiaca $\mathrm{e}$ dei generi minori rispetto alle più alte istanze della vita civile ${ }^{33}$, nonché dell'epica e della tragedia (e, come abbiamo visto, è lo stesso Marziale a utilizzare l'aggettivo in questo senso ${ }^{34}$ ). Se questo è vero, ci si potrebbe aspettare che (qualunque sia il senso di un'espressione cui Marziale sembra aver conferito un certo margine d'ambiguità) in 8,3 la Musa giochi, nel prosieguo della sua concione, sul significato che più comunemente si conferisce all'inerzia e alla pigrizia del poeta nei generi 'umili' : ed è esattamente quello che avviene. Talia sconsiglia al suo protetto di cimentarsi nell'epica o nella tragedia: le opere che si iscrivono in quei generi devono essere composte da autori gravi e severi (severi, v. 17), che, secondo l'antico topos neoterico e augusteo (e, del resto, di ascendenza già alessandrina), si affaticano a scrivere e a limare versi di notte (v. 18 : basti qui ricordare i poemi di Arato multum invigilata lucernis, 'molto vegliati a lume di lampada', di Cinna, fr. 11,1 Mor. / Bläns. / Courtn.). Insomma, la tragedia è genere da poeti operosi, non desidiosi: Marziale dovrebbe abbandonare i suoi otia epigrammatici, che gli hanno già assicurato la gloria (vv. 7-8), per affannarsi inutilmente in un genere vuoto, tronfio e, soprattutto, faticoso... Al tema dell'otium si associa indissolubilmente anche l'immagine 'bucolica' ai vv. 22-23, nei quali la Musa esorta il poeta a continuare a cantare con la sua gracile, 'bucolica' canna (angusta ...

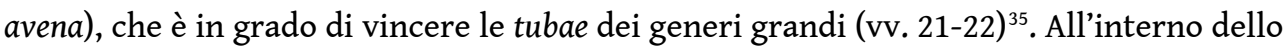
stesso libro 8, al c. 3 si riconnette il c. 55, dove Marziale torna a ricordare e a rimpiangere il ruolo di Mecenate per la poesia di Virgilio, per poi concludere, però (vv. $23-24)^{36}$ :

Ergo ego Vergilius, si munera Maecenatis des mihi ? Vergilius non ero, Marsus ero.

Sarò quindi Virgilio, se tu i regali di mecenate mi darai? Non sarò Virgilio, sarò Marso.

10 Anche se Marziale avesse un Mecenate, egli non diverrebbe un Virgilio, un poeta nel genere sublime dell'epos; egli sarebbe piuttosto un Domizio Marso, cioè il poeta epigrammatico più importante dell'età augustea ${ }^{37}$. Insomma, nel confronto tra il libro I e il libro VIII (da alcuni studiosi visto come un nuovo inizio dell'attività di epigrammista da parte di Marziale, dopo la pubblicazione di un primo corpus di sette libri numerati ${ }^{38}$ ), Marziale rivendica questa volta con orgoglio il proprio otium, che gli ha assicurato esattamente quella gloria poetica che avrebbe dovuto dargli il labor nei generi elevati in $1,107,5-8$; sicché, in 8,55, egli continuerebbe a dedicarsi all'epigramma anche se avesse in dono gli otia che Ottaviano e Mecenate diedero a Virgilio. Proprio in considerazione, però, dell'ormai ampia produzione e della gloria poetica che Marziale ha raggiunto, la dialettica otium-desidia vs labor si complica, come annuncia, ancor prima dell'ambiguo desidiosus in $8,3,12$, lo stesso proemio del libro VIII. Motivo 
adulatorio nei confronti del princeps è che, stavolta, il poeta ha dovuto 'penare' e faticare di meno con il suo ingenium, perché la materia offriva ampie risorse per fare poesia (minus itaque ingenio laborandum fuit, in cuius locum materia successerat, "ho dovuto lavorar meno con il mio talento naturale, in luogo del quale era subentrata l'altezza dell'argomento ${ }^{39}$. I sette libri si presentano supplici a Domiziano, cui devono la loro fama, ma hanno ormai un prestigio tale da poter fungere da patroni, presso l'imperatore, in favore del loro ottavo fratello. Dopo che Silvia Mattiacci ha sottolineato giustamente come Marziale rifiuti non tanto l'elaborazione formale in se stessa, quanto quella in generi 'alti', sentiti ormai come vacui ${ }^{40}$, Elena Merli ha ben analizzato il tema della lima in Marziale, arrivando a concludere correttamente che il recupero del tema in riferimento alla propria scrittura poetica avviene solo in singoli, rari momenti e con modalità molto peculiari, rispetto all'ormai tradizionale repertorio callimacheo o oraziano ${ }^{41}$. Se si considera questo quadro generale, la dialettica ingenium / materia che si instaura all'inizio dell'VIII libro sembra implicitamente esaltare il ruolo dell'ingenium nel successo dei primi sette libri marzialiani, mentre la materia 'imperiale' ha dato forma e sostanza all'ottavo ; in questa polarità sono esattamente l'ars e la lima che vengono meno, sono celate 'illusionisticamente' e non sono tematizzate. In modo significativo, il tema del labor viene 'spostato' dall' ars all' ingenium, dall'ambito dell'elaborazione formale a quello dell'ispirazione'. Io credo che Marziale si stia riconnettendo al tema, largamente sviluppato dall'Ovidio delle opere dell'esilio, della 'crisi dell'ingenium', capovolgendone il segno, ma mantenendo sostanzialmente il tema del rifiuto (o 'abbandono') della lima ${ }^{42}$. Altro aspetto rilevante ai nostri fini è che, fin dall'epistola prefatoria e poi nel carme 1 del libro VIII, il poeta è sicuro che l'epigramma possa celebrare degnamente l'imperatore e proprio per questo egli sottopone il suo libro ad una sorta 'purificazione rituale' prima di entrare nel tempio in cui si celebrerà Domiziano, vale a dire all'eliminazione della lasciva 'licenza' verbale tipica del mimo; presso i sacri penati dell'imperatore Venere nuda sarà bandita e sarà ammessa solo la Pallade cesariana ${ }^{43}$. Come si vede, la movenza è esattamente uguale e contraria rispetto a quella che abbiamo riscontrato in Priap. 2.

11 L'otium ha però in Marziale un altro risvolto, nel rapporto tra il poeta e il suo pubblico. L'ego auspica che il lettore interno 'illustre' possa dedicare i suoi otia, tra le sue mille occupazioni importanti, alla lettura dei libelli epigrammatici che gli vengono inviati : cfr. 4,14,10 ; 4,82,1-2 hos quoque commenda Venuleio, Rufe, libellos, / inputet et nobis otia parva roga, 'raccomanda anche questi libri a Venuleio, o Rufo, / e chiedigli di dedicarci un poco del suo tempo libero'; cfr. 11,1 etc. ${ }^{44}$ Ma il libellus è anche il 'beniamino' della plebe oziosa dell'Urbe, quanto mai indicato per essere il grazioso trastullo (deliciae) dei Quiriti : essi ne godranno negli otia tuta $(12,4,3)$ che il principe magnanimo ha offerto al popolo di Roma, secondo l'antico e ormai logoro motivo cortigiano inaugurato da Virgilio $^{45}$. Si tratta spesso, ad ogni modo, degli ozi di cui si gode in un determinato periodo dell'anno, 'liberato' dalle cure e dagli affanni, dai negotia consueti, uno spazio sacro che si può dedicare alla lettura delle nugae epigrammatiche. È la festa dei Saturnali, periodo propizio per questo genere di attività sia per il popolo (11,3,1-2 non urbana mea tantum Pipleïde gaudent / otia, nec vacuis auribus ista damus, "non solo $\mathrm{i}$ fannulloni dell'Urbe godono della mia Pipleide, né questi miei versi sono da noi offerti a orecchie da sfaccendati': interessante notare come Marziale, in realtà, affermi che il suo liber ormai è diffuso nell'orbis intero, persino tra i militari in Dacia o in Britannia, i più lontani dal potersi permettere gli ozi romani...), sia per il patrono illustre (cfr. 7,28,7-8 otia dum medius praestat tibi parva December, / exige, sed certa, quos legis, aure iocos, a Fusco: 
'mentre Dicembre nel mezzo ti offre brevi ozi, / esamina questi scherzi letterari che leggi, ma con orecchio attento'): l'imperatore è una sorta di patronus dei Saturnalia, soprattutto in un libro come l'XI (11,2,5-6 : clamant ecce mei 'Io Saturnalia' versus : / et licet et sub te praeside, Nerva, libet, 'gridano, ecco, i miei versi 'hurrà, Saturnali!' / è lecito e, sotto il tuo patrocinio, piacevole, o Nerva' ${ }^{46}$. È però l'Urbe in persona, con il pilleus, a dare licenza perché, in questo spazio franco della festa, si possa 'scherzare con verso che non costa fatica', versu ludere non laborioso (11,6,3: pur nelle differenze di contesto, motivi e linguaggio presentano innegabili analogie con Priap. 2,3 scripsi non laboriose, in riferimento ai versi scritti nello spazio sacro del dio).

L'attività di scrittura e di lettura avvengono 'in fretta' : si assiste al superamento del topos del labor limae, la festinatio nel lavoro del poeta diviene da un lato caratteristica di cui scusarsi con il patrono, dall'altro affermazione di abilità e dignità di letterato 'professionista', di fronte ai dilettanti 'oziosi' o ai patroni 'impegnati', che leggono in fretta ciò che in fretta è stato scritto ${ }^{47}$. Fin dal Liber Spectaculorum 31 Lindsay $(=35$ Coleman ${ }^{48}$ ), Marziale invita il Caesar a perdonarlo per i suoi versi scritti in fretta, perché non merita di risultare sgradito colui che si affretta ad essere gradito (da veniam subitis: non displicuisse meretur, / festinat, Caesar, qui placuisse tibi, 'scusa queste improvvisazioni: non merita di dispiacerti, / o Cesare, chi si affretta a piacerti'). Festinat poeta, ma festinat anche il lector: Marziale arriva a scusarsi perché il suo libellus (il XII) è stato assemblato in pochi giorni per l'arrivo in Spagna del patrono Prisco e a chiedergli di farsi garante, con una lettura attenta, affettuosa ma critica, di questo libro messo insieme in modo un po' trafelato ${ }^{49}$; tranne poi, nel primo epigramma del libro stesso, assicurare Prisco del fatto che il libellus può essere letto in quattro e quattr'otto (hora non aestiva...), non tanto nelle pause delle attività politiche o amministrative, ma addirittura negli intervalli (otia !) di una partita di caccia ${ }^{50}$. La contraddizione tra i due brani, uno di seguito all'altro, sembra davvero studiata : vi è una dinamica sottile, nei libelli marzialiani, tra lettura 'impegnata' e lettura 'oziosa' dei patroni, nella quale spesso l'autore dà fondo alle sue risorse d'arguzia ${ }^{51}$. Ma, come dicevo sopra, tutti i motivi che abbiamo visto vanno inquadrati all'interno di quella complessa dinamica tra ozio e letteratura, festinatio del poeta professionista e 'fretta' del lettore: ne risulta esaltata l'abilità di poeta che compone rapidamente per l'occasione (l'inaugurazione del Circo Massimo, l'arrivo del patrono in Spagna...), ma è altresì in grado di pubblicare libelli di così elevata qualità che verranno letti toto ... orbe, 'in tutto il mondo', (Mart. 1,1,2 toto notus in orbe), in cui ogni lettore, anche il più distratto, il più superficiale e frettoloso, potrà trovare quello che lo soddisfa (si arriva alla satira di questo atteggiamento già in 14,2,3-4 : lemmata si quaeris cur sint adscripta, docebo: / ut, si malueris, lemmata sola legas, 'mi chiedi perché siano posti i titoli, e te lo dirò: / perché, se preferirai, tu legga solo i titoli') ${ }^{52}$.

Otiosus è in Marziale sia il patrono Canio Rufo, letterato finissimo e poliedrico, quando si trastulla con l'epigramma piuttosto che con i generi elevati $(3,20,8-9$ an otiosus in schola poetarum / lepore tinctos Attico sales narrat?, 'forse che ozioso nel circolo dei poeti / sta recitando versi pieni di spirito, tinti d'arguzia d'Attica?': il linguaggio è neoterizzante ${ }^{53}$ ), sia addirittura il libellus stesso del poeta, nel momento in cui vaga 'sfaccendato' in cerca di lettori, conciato come un damerino, secondo un inveterato topos di ascendenza oraziana (è ancora il 'saturnalizio' libro XI, 'ozioso' e non adatto all'impegnatissimo, illustre Partenio, bensì alla turba otiosa del portico di Quirino: 11,1-2 quo tu, quo, liber otiose, tendis / cultus Sidone non cotidiana ?, 'dove, dove ti dirigi, libro ozioso, / decorato di porpora non ordinaria?') $)^{54}$. 


\section{Otium e letteratura nei Carmina Priapea.} possiamo osservare che la dinamica tra ozio e letteratura, all'interno di Priap. 1,2, allude sì a Catull. 1, ma perché il lettore sia attento alla sottile ridefinizione degli aspetti metapoetici. Poesia 'nugatoria', apparentemente disimpegnata ed eleganza del libellus sono aspetti complementari in Catullo: nel proemio dei Priapea si arriva invece al paradosso di affermare che i versi priapici non sono neppure adatti ad un liber di poesia, elegante o meno che sia. I carmi sono appropriati al giardino di Priapo $(2,2)$ e sono scritti non su raffinato volumen di papiro, bensì su supporto ben diverso : essi si collocano in uno spazio sacro e 'liberato' come lo erano i Saturnali o la festa della dea Flora (Mart. 1 praef. ; 1,35) per Marziale, e cioè le pareti del tempio di Priapo (2,9-10). Io credo che questo plateale gesto con cui l'autore si sottrae al liber per rifugiarsi nello spazio sacro a Priapo vada letto tenendo presente non solo l'estetica del libro in Catullo e in Marziale, ma anche il secondo proemio di Stratone, laddove si parla, comunque, di

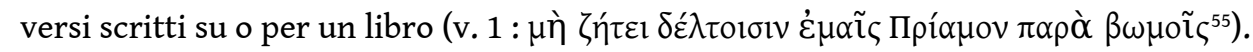

L'anonimo autore dei Priapea elimina quel nesso tra otium dell'autore e libro di letteratura (manufatto, comunque, raffinato e 'urbano') che Marziale continuava ad affermare (ed anzi, sviluppava ulteriormente). Affidandosi alla protezione del dio Priapo, egli 'smaschera' quell'atteggiamento che lasciava convivere futilità di contenuti, esibito 'disimpegno' e qualità dei versi, frutto di abilità del poeta riconosciute da un pubblico e garantite dalla diffusione di libelli. Scrivere versi 'per gioco' (2,1 ludens), da otiosus, significa comporre carmi 'improvvisati' o francamente 'rozzi' (Priap. 1,1 carminis incompti), inadeguati al libro : c'è un'istanza realistica che il poeta afferma nell'attribuire al lusus e all'otium (o meglio, all'essere otiosi) dei significati radicali che la poetica del 'trastullo raffinato' o comunque 'letterario' inaugurata dai neoterici aveva cancellato o sublimato. Certo, qualcosa di simile, pur con tutte le grandi differenze del caso, era già in Catull. 50, e non è da escludere che suggestioni arrivino proprio da quel carme ${ }^{56}$. Catullo vi ricordava i versi ludici e improvvisati che egli aveva composto in gara con Calvo (vv. 1-2 hesterno, Licini, die otiosi / multum lusimus in meis tabellis, 'ieri, Licinio, durante il giorno, sfaccendati, / giocammo a lungo sulle mie tavolette ${ }^{57}$ ). Sono carmi composti in allegria (v. 6 in ioco atque vino, 'tra vino e scherzi'), escogitati in promptu e trascritti sulle tavolette, ma che non prevedono certo una pubblicazione su libro: e infatti Catullo fa avvertire al lettore la differenza che esiste tra quei versiculi improvvisati oziosamente (dei quali non riporta neppure una parola) e il poema di cui parla al v. 16 (il carme 50 stesso o, secondo altri, il successivo carme 51), che invece leggiamo ancora oggi nel Liber... carme 1 il poeta 'presenta' al lettore la sua poesia improvvisata ponendolo di fronte alla statua del dio : è assurdo leggere con severità quei versi licenziosi, significherebbe voler coprire con una veste il membro nudo di Priapo. L'immagine è chiaramente ispirata all'incipit del I libro di Marziale (praef. : non intret Cato theatrum meum, aut si intraverit, spectet, 'non entri Catone nel mio teatro, o se è entrato, guardi'), nonché a quella di 1,35, in cui i grammatici severi vorrebbero che i versi di Marziale fossero privati della loro salacità : sarebbe come rivestire le nude, procaci attrici che si esibiscono nella festa di Flora (vv. 8-9 quis Floralia vestit et stolatum / permittit meretricibus pudorem ?, 'chi è che 
impone vestiti alle feste di Flora e alle meretrici il pudore della stola?') ${ }^{58}$. Ma c'è qualcosa di diverso che, a ben interpretarlo, dà ragione delle caratteristiche peculiari di questa raccolta rispetto ai modelli epigrammatici latini. L'ego poetico non accoglie il lettore nel 'suo' teatro (theatrum meum), come faceva in Marziale, né gli offre un elegante libretto da leggere nel tempo sacro dei Saturnali. Egli, piuttosto, nel dittico iniziale della raccolta, lo 'accompagna', nel suo girovagare da ozioso, all'interno del tempio di Priapo (e ne lascia fuori le Muse...) : insomma, egli si pone non di fronte al lettore, ma al suo fianco, all'interno dello spazio consacrato di questa nuova poesia, a contemplare insieme a lui l'enorme mentula del dio. Al pari di un qualunque sfaccendato, di uno scariphator perditempo, egli entra nel tempio di Priapo e graffisce i suoi ruvidi versi. Come è stato ben notato, c'è una 'autodegradazione' della poesia epigrammatica a incondito 'graffito', una messa in scena che ben veicola e introduce l'oscenità dei contenuti ${ }^{59}$; anche questo motivo è già marzialiano $0^{60}$. Una conseguenza importante è che, nel gioco che viene proposto al lettore, la funzione, l"aura' del poeta esce depotenziata ${ }^{61}$, o meglio, sembra distribuirsi tra i diversi soggetti, parimenti oziosi, che popolano il tempio/giardino del dio : fatto sta che l'ego poetico che parla nei primi due carmi sostanzialmente scompare nel prosieguo della raccolta e a parlare sono il dio stesso (soprattutto) e coloro che entrano nello spazio da lui controllato. Frequenti sono gli epigrammi di dedica a Priapo, da parte di varie figure più o meno maliziose : e il primo dedicante è l'ego poetico stesso nel carme 2, in cui, più che essere 'ispirato' dal dio, egli chiede la sua testimonianza e gli offre in dono i suoi stessi versi. La parola, subito dopo, sarà ceduta al dio, che esporrà, nel carme 3 , la sua rozza poetica che non prevede alati riferimenti al mito o alla dottrina filologica, ma il linguaggio schietto e osceno confacente al suo fallo smisurato : e il dio parla davvero, ormai, come un retore o un poeta, compiaciuto, lodando la sua 'ispirazione' tutt'altro che sublime $(3,10$ crassa Minerva mea est, 'la mia Minerva è crassa') ${ }^{62}$. Sulla scia del carme 1, si manifesta pienamente un aspetto complementare a quelli fin qui analizzati, e cioè la rusticitas di Priapo: paradossalmente, però, essa che è in genere contrapposta all'urbanitas cittadina, meglio realizza proprio quegli ideali di 'semplicità' e purezza, di Latinitas (simplicius multo est 'da pedicare' Latine / dicere, 'è di molto più semplice dire 'dammi da inculare', alla latina'), che sono le caratteristiche della lingua dell'epigramma romano secondo Marziale (cfr. ad es. $11,20^{63}$ ). Subito dopo, in questa sequenza iniziale perfettamente congegnata (anche nell'alternanza dei metri e dei formati epigrammatici ${ }^{4}$ ), la parola passa alla prima delle figure di dedicante (carme 4), una ragazza che offre delle tavole su cui sono rappresentate figure erotiche, con i vari schemi di accoppiamento ricavati 'dai libri' della famosa scrittrice Elefantide (v. 2 ex Elephantidos libellis). La ragazza chiede che Priapo faccia sì che ella 'metta in pratica' (opus edat) quegli schemata erotici di cui ella offre al dio la rappresentazione in figura ${ }^{65}$ : se si eccettua l'eloquente 68,2 (libros non lego, poma lego, 'non leggo libri, raccolgo frutti' $\left.{ }^{66}\right)$, è l'unica volta in cui si parlerà nuovamente di libri e libelli nel corso della raccolta $\mathrm{e}$ la loro funzione è, come si vede, degradata (non si offre al dio dotta poesia erotica di stampo alessandrino, anzi, propriamente non si offre neppure poesia, bensì i quadretti che illustrano gli scritti di un'autrice ben nota, più che altro, per la sua licenziosità). La richiesta della dedicante è esplicita e diretta, all'insegna di quel realismo salace che è la cifra di tutta la raccolta. Alla fine della sequenza iniziale, nel c. 5 il dio prende di nuovo la parola per imporre la sua legge (lex), per chi vorrà entrare nel suo spazio sacro, in una comica lingua giuridico-sacrale ${ }^{67}$, e nei termini di un do ut des dai connotati sessuali che verrà ripreso e (solo in parte) ribaltato dal successivo c. $41^{68}$. In quest'ultimo 
componimento, ognuno dei dedicanti che entrerà nel tempio/giardino diventerà a sua volta 'poeta': nello spazio liberato di Priapo ognuno esprime liberamente, giocosamente, oziosamente, la sua salacità, sull'esempio del poeta nel carme 2. Questo è il senso del carme 41 (che sarà poi ulteriormente chiarito a breve distanza dai cc. $47 \mathrm{e}$ 49), senza alcun dubbio un vero e proprio 'proemio in mezzo' : quisquis venerit huc, poeta fiat / et versus mihi dedicet iocosos. / qui non fecerit, inter eruditos / ficosissimus ambulet poetas, 'chiunque viene qui, diventi poeta / e mi dedichi versi giocosi. / Chi non lo fa, vada in giro tra poeti eruditi, / tutto pieno di fistole'). Priapo in persona detta le regole nel suo piccolo regno, all'insegna di un allegro anti-intellettualismo: chi le rifiuta andrà a 'passeggiare' (ambulet) tra poeti eruditi, dopo aver subito la pesante vendetta del dio (ficosissimus) ${ }^{69}$.

La strategia comunicativa propone una sistematica 'degradazione' dei tratti essenziali della poetica non solo neoterica, ma anche marzialiana: non solo il gusto per l'erudizione si muta in crassa Minerva, ma l'urbanitas si deforma in rozzezza rustica, l'ozio operoso del poeta diviene un poltrire da bighellone; la funzione autoriale ne esce indebolita, ma ne risulta sconvolta anche la stessa funzione del lettore, non più complice del poeta dotto come in Catullo, né ozioso cittadino, esigente consumatore di libelli o patrono più o meno 'affaccendato' come in Marziale, bensì spettatore della verga di Priapo, comunque accanto al poeta, e in fondo poeta anche lui ${ }^{70}$. Il dispositivo iniziale vuole creare il presupposto per una raccolta in cui domini la voce non di un ego poetico, ma dello stesso rusticus dio, nonché di una serie di personae che via via entrano ed escono dallo spazio consacrato di Priapo (e della poesia). Il liber di poesia viene bandito da questo sistema così radicalmente ridefinito : ma si sbaglierebbe a pensare ad un reale sovvertimento degli elementi fondativi del genere epigrammatico in Roma. Nonostante l'ostentato rifiuto del libro, in fondo, i lettori 'priapici' leggeranno questi carmi su un libellus abilmente costruito e confezionato, dall'inizio alla fine ${ }^{71}$; e negli studi sull'opera comincia finalmente a prevalere l'idea che un simile raffinato dispositivo librario e una così attenta rivisitazione delle convenzioni del genere epigrammatico latino sono impensabili se non ammettendo che i Priapea sono opera di un unico poeta, già largamente influenzato dalla poetica marzialiana ${ }^{72}$. La poesia nugatoria vuole, da sempre, realizzare un ideale di lepos, di sapida arguzia, persino di fronte alla materia più refrattaria, e nelle condizioni più estreme : i Priapea non fanno che obbedire ancora a questa istanza, nel modo più paradossale, giocando a portare quella poetica agli estremi, oltre i quali essa si dissolve. Per la sua finzione letteraria, l'autore costruisce sempre il suo theatrum e desidera sempre la complicità del lettore : sarebbe ingiusto se gliela negassimo.

\section{BIBLIOGRAFIA}

Baehrens, Ae. (ed.), 1879 : Poetae Latini Minores, I, Lipsiae. 
Barchiesi, A., 2001 : Genealogie letterarie nell' epica imperiale : fondamentalismo e ironia, in E.A. Schmidt (hrsg.), L'histoire littéraire immanente dans la poésie latine, Fondation Hardt, Entretiens sur l'Antiquité classique, 47, Genève - Vandœuvres, 315-354.

Bellandi, F., 1995 : L'immagine di Mecenate protettore delle lettere nella poesia fra I e II sec. d.C., "A \& R" n.s. $40,78-101$.

Biville, F. - Plantade, E. - Vallat, D. (éd. par), 2008 : « Les vers du plus nul des poètes... ». Nouvelles recherches sur les Priapées, Actes de la journée d'études organisée le 7 novembre 2005 à l'Université Lumière-Lyon 2, Maison de l'Orient et de la Méditerranée - Jean Pouilloux, Lyon.

Bowie, M.N.R., 1988 : Martial Book XII. A Commentary, Diss. Oxford.

Buchheit, V., 1962 : Studien zum Corpus Priapeorum, München.

Buchheit, V., 2007 : Einheit und Zeit der Carmina Priapea, "Hermes” 135, 74-79.

Buecheler, F. (ed.), $1882^{3}$ : Petronii Satirae et Liber Priapeorum, Berolini.

Callebat, L., 2008 : Les Priapées : éléments d'une problematique, in Biville - Plantade - Vallat 2008, 23-32.

Callebat, L. (texte ét. trad. et comm. par), 2012 : Priapées, Paris.

Cano, P.L., - Velázquez, J. (ed.), 2000 : Carmina Priapea : a Príapo, dios del falo, Bellaterra (Barcelona).

Canobbio, A., 2005 : Il libro VIII di Marziale e la ricerca di una identità augustea, in F. Gasti - G. Mazzoli (a cura di), Modelli letterari e ideologia nell'età flavia.Atti della III Giornata Ghisleriana di Filologia classica, Como - Pavia, 127-162.

Canobbio, A., 2014 : Generi 'grandi' e generi 'piccoli' in Marziale e in Stazio, "BSL" 44, 442-470.

Cazzaniga, E. [I.] (ed.), 1959 : Carmina ludicra Romanorum, Augustae Taurinorum.

Citroni, M., 1968 : Motivi di polemica letteraria negli epigrammi di Marziale, "DialArch" 2, 259-301.

Citroni, M., 1975 : M. Valerii Martialis Epigrammaton liber I, Introduzione, testo, apparato critico e commento, Firenze.

Citroni, M., 1989 : Marziale e la letteratura per i Saturnali (poetica dell'intrattenimento e cronologia della pubblicazione dei libri), "ICS” 14, 201-226.

Citroni, M., 2008 : Les proèmes des Priapées et le problème de la datation du recueil, in Biville - Plantade - Vallat 2008, 35-51.

Codoñer Merino, C. - González Iglesias, J.A., 2015 : Priapea : introducción y edición crítica, C. C. M. ; traducción, notas y comentario literario, J. A. G. I., Huelva.

Coleman, K.M., 1988 : Statius. Silvae IV. Edited with an English Translation and a Commentary, Oxford.

Coleman, K.M., 2006 : Martial. Liber Spectaculorum. Edited with Introduction, Translation and Commentary, Oxford.

Craca, C., 2011 : Dalla Spagna : Gli epigrammi 1-33 del XII libro di Marziale, Bari.

Eickhoff, F.C. (hrsg.), 2016 : Muße und Rekursivität in der antiken Briefliteratur. Mit einem Ausblick in andere Gattungen, Tübingen.

Dau, A., 1887 : De Marci Valerii Martialis libellorum ratione temporibusque, Diss. Rostochii.

De Miguel Mora, C., 2008 : Catulo en los Carmina Priapea, in Biville - Plantade - Vallat 2008, 83-98.

Degl'Innocenti Pierini, R., 1999 : Tra filosofia e poesia. Studi su Seneca e dintorni, Bologna. 
Fiorucci, F. (hrsg.), 2017 : Muße, otium, $\sigma \chi 0 \lambda \eta ́$ in den Gattungen der antiken Literatur, Freiburg et al.

Floridi, L. (testo critico, traduzione e commento a cura di), 2007 : Stratone di Sardi. Epigrammi, Alessandria.

Fountoulakis, A., 2013 : Male bodies, male gazes : exploring Erôs in the twelfth book of the 'Greek anthology', in E. Sanders et al., Erôs in ancient Greece, Papers from a conference held at University College London in 2009, Oxford-New York, 293-312.

Fowler, D.P., 1995 : Martial and the Book, "Ramus" 24, 31-58.

Fusi, A., 2006 : M. Valerii Martialis Epigrammaton liber tertius. Introduzione, edizione critica, traduzione e commento, Hildesheim.

Goldberg, Chr., 1992 : Carmina Priapea. Einleitung, Übersetzung, Interpretation und Kommentar, Heidelberg.

Gómez Pallarès, J., 1995 : Cuestiones en torno al 'otium' en Marcial, “RPL” 18, 61-89.

Hallett, J.P., 1996 : Nec castrare velis meos libellos : Sexual and Poetic Lusus in Catullus, Martial and the Carmina Priapea, in C. Klodt (hrsg.), Satura Lanx. Festschrift für Werner A. Krenkel zum 70. Geburtstag, Hildesheim - Zürich - New York, 321-344.

Heraeus, W., $1976^{2}$ : M. Valerii Martialis epigrammaton libri, Editionem correctiorem cur. I. Borowskij, Lipsiae.

Höschele, R., 2008a : Priape mis en abyme ou comment clore le recueil, in Biville - Plantade - Vallat 2008, 53-66.

Höschele, R., 2008b : Longe longissimum. Il carmen 68 del Corpus Priapeorum, in Morelli 2008c, 383-396.

Höschele, R., 2010 : Die blütenlesende Muse. Poetik und Textualität antiker Epigrammsammlungen, Tübingen.

Holzberg, N., 2002 : Martial und das antike Epigramm, Darmstadt.

Holzberg, N., 2005 : Impotence ? It happened to the best of them ! A Linear Reading of the Corpus Priapeorum, "Hermes" 133, 368-381.

Howell, P., 1980 : A Commentary on Book One of the Epigrams of Martial, London.

Izaac, H.J., 1933 : Martial. Épigrammes. Texte établi et traduit, II, Paris.

Kay, N.M., 1985 : Martial, Book XI. A Commentary, London.

Kißel, W., 1990 : Persius Flaccus, Aules. Satiren. Herausgegeben, übersetzt und kommentiert, Heidelberg.

Kloss, G., 2003 : Überlegungen zur Verfasserschaft und Datierung der Carmina Priapea, "Hermes" 131, 464-487.

Lhommé, M.-K., 2008 : Constructions cultuelles dans les Priapées : la séquence centrale Pr. 40-42, in Biville - Plantade - Vallat 2008, 139-155.

Lindsay, W.M. (ed.), $1929^{2}\left(1903^{1}\right)$ : M. Valeri Martialis Epigrammata, Oxonii.

Lorenz, S., 2002 : Erotik und Panegyrik. Martials epigrammatische Kaiser, Tübingen.

Maggi, A., 1923 : I Priapea. Revisione del testo e commento, Napoli.

Maltby, R., 2002 : Tibullus, Elegies. Text, Introduction and Commentary, Cambridge. 
Mattiacci, S., 2007a : Marziale e il neoterismo, in A. Bonadeo - E. Romano (a cura di), Dialogando con il passato, Firenze, 177-206.

Mattiacci, S., 2007b : Marziale e la fortuna del neoterismo nella prima età imperiale, in Ead. - A. Perruccio, Anti-mitologia ed eredità neoterica in Marziale : genesi e forme di una poetica, Ospedaletto, 135-218.

Mattiacci, S., 2016 : I lascivi versus di Augusto citati da Marziale e la tecnica dell'epigramma nell'epigramma, in B. Pieri - D. Pellacani (a cura di), Si verba tenerem. Studi sulla poesia latina in frammenti, Berlin - Boston, 111-132.

Merli, E., 1993 : Ordinamento dei epigrammi e strategie cortigiane negli esordi dei libri I-XII di Marziale, "Maia" 45, 229-257.

Merli, E., 2006 : Martial between Rome and Bilbilis, in R.M. Rosen - I. Sluiter (ed. by), City, Countryside, and the Spatial Organization of Value in Classical Antiquity, Leiden - Boston, 327-347.

Merli, E., 2013 : Dall'Elicona a Roma : acque ispiratrici e lima poetica nell'Ovidio dell'esilio e nella poesia flavia di omaggio, Berlin.

Mondin, L., 2011 : Catullo 1 e Meleagro, in A. Balbo - F. Bessone - E. Malaspina (a cura di), 'Tanti affetti in tal momento'. Studi in onore di Giovanna Garbarino, Alessandria, 659-672.

Morelli, A.M., 2008a : recensione a Biville - Plantade - Vallat 2008, “RPh” 82, 527-531.

Morelli, A.M., 2008b : Epigramma longum : in cerca di una basanos per il genere epigrammatico, introduzione a Morelli 2008c, 17-51.

Morelli, A.M. (a cura di), 2008c : Epigramma longum. Da Marziale alla tarda antichità / From Martial to Late Antiquity, Atti del Convegno internazionale, Cassino, 29-31 maggio 2006, Cassino.

Morelli, A.M., 2016 : Calvus et l'image du poète dans l'œuvre de Catulle (à propos des thèmes du banquet en Catull. 50), in L. Foschia - E. Santin (éd. par), L'épigramme dans tous ses états : épigraphiques, littéraires, historiques, Lyon, 247-277 (openedition book : https://books.openedition.org/ enseditions/5621).

Morelli, A.M., 2017 : Entre le petit et le ridicule. Pour une histoire comparée de l'épigramme satirique grecque et latine, in D. Meyer - C. Urlacher-Becht (éd. par), La rhétorique du petit dans l'épigramme grecque et latine, Actes du colloque de Strasbourg (26 et 27 mai 2015), Paris, pp. 131-147.

Morelli, A.M., 2018 : Un simposio per le acque. Stazio e il balneum di Claudio Etrusco (Silu. 1,5), "SIFC" n.s. $16,71-101$.

Moreno Soldevila, R., 2006 : Martial, Book IV. A Commentary, Leiden - Boston.

Moretti, G., 2010 : Xenia e Apophoreta di Marziale tra ekphrasis retorica e tradizione iconografica della 'natura morta', in L. Belloni, A. Bonandini - G. Ieranò - Ead. (a cura di), Le Immagini nel Testo, il Testo nelle Immagini : rapporti fra parola e visualità nella tradizione greco-latina, Trento, 327-372.

Myers, K.S., 2015 : Statius on Invocation and Inspiration, in W.J. Dominik - C.E. Newlands - K. Gervais (eds.), Brill's Companion to Statius, Leiden, 31-53.

Nauta, R.R., 2002 : Poetry for Patrons. Literary Communication in the Age of Domitian, Leiden et al. Nauta, R.R., 2006 : The recusatio in Flavian Poetry, in Id. - H.J. Van Dam - J.J.L. Smolenaars (ed. by), Flavian Poetry, Leiden - Boston, 21-40.

Neger, M., 2012 : Martials Dichtergedichte. Das Epigramm als Medium der poetischen Selbstreflexion, Tübingen. 
Nisbet, R.G.M., Hubbard, M., 1978 : A Commentary on Horace : Odes, Book II, Oxford.

Pérez Vega, A. - Ramírez De Verger, A. (ed. por), 2005 : C. Valerii Catulli Carmina - Catulo : Poemas, Huelva.

Pitcher, R.A., 1998 : Martial's debt to Ovid, in F. Grewing (hrsg.), Toto notus in orbe : Perspektiven der Martial-Interpretation, Stuttgart, 59-76.

Plantade, E., 2008 : Priapus gloriosus. Poétique d'un discours compensatoire, in Biville - Plantade Vallat 2008, 99-119.

Prioux, É., 2008 : At non longa bene est ? Priape face à la tradition du discours critique alexandrin, in Biville - Plantade - Vallat 2008, 157-180.

Richlin, A., $1992^{2}$ : The Garden of Priapus. Sexuality and Aggression in Roman Humor, New YorkOxford.

Rimell, V., 2008 : Martial's Rome. Empire and the Ideology of Epigram, Cambridge.

Roman, L., 2014 : Poetic Autonomy in Ancient Rome, Oxford - New York.

Rosati, G., 2002 Muse and Power in the Poetry of Statius, in E. Spentzou - D. Fowler (ed. by), Cultivating the Muse. Struggles for Power and Inspiration in Classical Literature, Oxford, 229-251.

Scherf, J., 2001 : Untersuchungen zur Buchgestaltung Martials, München - Leipzig.

Schöffel, Chr., 2002 : Martial, Buch 8. Einleitung, Text, Übersetzung und Kommentar, Stuttgart.

Socas, F. 2004 : 'Lemmata sola legas' : una revisión de "Xenia » y « Apophoreta », in J.J. Iso Echegoyen A. Encuentra Ortega (ed. por), Marcial, 1900 años después, Zaragoza, 227-246.

Solaro, G., 1993 : La dedica catulliana e il secondo proemio dei Priapea, "Sileno" 19, 533-538.

Sparagna, S., 2014 : L'unità del XII libro degli Epigrammi di Marziale, Diss. Dott. Cassino.

Sparagna, S., 2016 : Il ciclo della clientela nel XII libro degli Epigrammi di Marziale (18, 29 [26], 57, 68), "Eikasmos" 26, 153-176.

Thomson, D.F.S., 1997 : Catullus. Edited with a Textual and Interpretative Commentary, Toronto.

Tränkle, H., 1999 : Entstehungszeit und Verfasserschaft des Corpus Priapeorum, “ZPE” 124, 145-156.

Vallat, D., 2012 : Hors du jardin, hors de l'épigramme : pour une relecture métapoétique des Priapées, "BSL" 42, 15-28.

Vollmer, F. (ed.), 1923 : Poetae Latini Minores, II 2, Lipsiae.

Young, E., 2015 : Dicere Latine : the art of speaking crudely in the Carmina Priapea, in D. Dutsch - A. Suter (ed. by), Ancient Obscenities : Their Nature and Use in the Ancient Greek and Roman Worlds, Ann Arbor, 255-282.

\section{NOTE}

1. Una prima versione di questo testo (tradotto in spagnolo con sensibilità e competenza da Roberta Alviti, che ringrazio di cuore) è stata presentata al convegno SELAT Otium et negotium. El legado de Roma, a León, nel luglio 2016, in onore di Francisca Moya Del Baño. Le dedico senz'altro anche la versione che qui pubblico. Voglio esprimere qui la mia profonda gratitudine agli organizzatori, che mi hanno gentilmente invitato al convegno, a Mario Citroni e a José Carlos Miralles Maldonado, con cui ho discusso gli argomenti che qui tratto, ai direttori e al comitato 
scientifico della rivista, che ora accoglie l'articolo. Ringraziamenti sinceri anche ai due revisori anonimi per le loro osservazioni. Mia rimane la responsabilità per errori e omissioni.

2. Citroni 2008: ho sviluppato una parte delle riflessioni che propongo in queste prime pagine del mio intervento già in Morelli 2008a. Cfr. poi Höschele 2010, soprattutto 275-276 (e cfr. già la tesi dottorale dal medesimo titolo, München 2007).

3. Utilizzo il testo di Codoñer Merino - González Iglesias 2015, 124-127, ma ho tenuto presente anche Baehrens 1879, 58; Buecheler 1882³ , 137; Maggi 1923, 1-4; Vollmer 1923, 43; Cazzaniga 1959, 23-24; Cano - Velázquez 2000, 64-67; soprattutto, Callebat 2012, 2-3.

4. Citroni 2008.

5. Buchheit 1962, 3-13.

6. Per il testo di Marziale, prendo a base l'edizione di Lindsay $1929^{2}\left(1903^{1}\right)$; ho sistematicamente consultato anche Heraeus $1976^{2}$, nonché le edizioni parziali e i commenti via via citati in nota.

7. Citroni 2008, 46-47; cfr. poi Höschele 2010, 277, e vd. anche infra, § 4.

8. Citroni 2008, 42-43; cfr. anche infra, in questo stesso $\S$.

9. Cfr., in particolare, Citroni 2008, 43; Höschele 2010, 275-276. Quanto ai due carmi proemiali della raccolta stratoniana, adotto il testo di Floridi 2007, 58; cfr. anche il ricco commento, 117-123, con bibliografia precedente (sul confronto tra i due carmi proemiali stratoniani e Priap. 1-2, cfr. 6-7 e 119); si aggiunga almeno Fountoulakis 2013.

10. Buchheit 1962, 12-13; 109-112.

11. Cfr. ancora Citroni $2008,43$.

12. Cfr. Floridi 2007,122 , che fa notare il rapporto imitativo tra l'epigramma di Stratone e Meleag. AP 7. 419. 3-4 (HE 4003-4004), ove anche sono invocati Eros e le Cariti, ma insieme alle

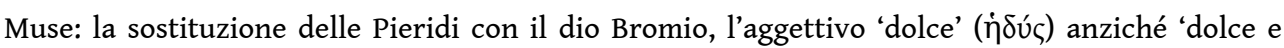
lacrimevole' ( $ү \lambda u \kappa v ́ \delta \alpha \kappa \rho u \varsigma)$ in riferimento all'amore, orientano verso una concezione disimpegnata dell'Eros simposiale, ove però si rinuncia al pathos e alla dottrina stucchevole, non certo all'eleganza formale.

13. Tali effetti sono spesso al centro di analisi raffinate all'interno degli studi proposti nel volume lionese in cui si inserisce lo studio di Citroni 2008 (vd. anche infra).

14. Cfr. almeno Stat. Silv. 1,4,19-30; 1,5,1-5; 1,6,1-7, 2,3,6-7, e 5,3,1-3, nella recente analisi di Myers 2015, 47-53 (cfr. già Rosati 2002, 245), mentre un po' diversi sono i casi di 4,7,21 (su cui cfr. anche Coleman 1988, 202-203, e Merli 2013, 92 e 187-189, per il rapporto con il tema iniziale del 'differimento' della poesia epica, del resto già presente in 1,5) e 5,1,13-15, che Myers 2015, 51-53, riconduce giustamente al motivo poetico dell'aporia in un caso e al ruolo di 'ispiratore' del princeps nell'altro. I precedenti del motivo nella letteratura latina sono, soprattutto, in ambito elegiaco (cfr. Prop. 2,1,1-4 e Ov. Ars 1,25-29, con le osservazioni ancora di Myers 2015, 48).

15. Catull. 1: Cui dono lepidum novum libellum / arida modo pumice expolitum? / Corneli, tibi: namque tu solebas / meas esse aliquid putare nugas / iam tum, cum ausus es unus Italorum [5] / omne aevum tribus explicare chartis, / doctis, Iuppiter, et laboriosis. / quare habe tibi quidquid hoc libelli, / qualecumque: quod, <0> patrona Virgo, / plus uno maneat perenne saeclo [10]. Adotto il testo di Pérez Vega Ramírez De Verger 2005, 90, tenendo presente anche Thomson 1997, 99. Come mi fa giustamente notare uno dei revisori anonimi, con l'espressione al v. 11 in partem accipias bonam il poeta dei Priapea riprende habe tibi in Catull. 1,8, ma modificandone in parte i significati: più che la modestia del dono rispetto ad un lettore illustre come Cornelio Nepote, ad essere in questione è soprattutto il carattere non offensivo e non 'sacrilego', nei confronti del dio, dei rozzi versi graffiti nel suo tempio.

16. A mio parere, il ricordo delle chartae laboriosae di Nepote, nel dispositivo proemiale e metapoetico creato da Catullo, si lega indissolubilmente alle allusioni a Meleagro (non solo e non tanto al proemio dello Stephanos, quanto soprattutto alla Coronide, AP 12,257,3, con l'idea di letteratura come 'fatica', $\mu$ óxӨo५: cfr. Mondin 2011, 664, con bibliografia precedente). 
17. Sul confronto tra Priap. 2 e Catull. 1, cfr. Buchheit 1962, 11; Goldberg 1992, 64; Solaro 1993; Hallett 1996, 336-337; Citroni 2008, 44; De Miguel Mora 2008, 91-94. Sul rapporto con Priap. 2,3, cfr. anche Höschele 2010, 281-282.

18. Naturalmente, non è possibile, nei limiti di questo saggio, affrontare una trattazione d'insieme del tema dell'otium nelle letterature d'epoca classica, oggetto di recente ancora di ampie discussioni e di importanti opere miscellanee: segnalo quella complessiva di Fiorucci 2017 e quella, più specifica, ma non priva di risvolti importanti anche per altri generi letterari, di Eickhoff 2016 (la miscellanea contiene contributi sull'epistolografia sia in prosa sia in poesia: considerata l'importanza della forma del $\gamma \rho \alpha \mu \mu \alpha \tau i ́ \delta 1 o v$ o comunque di talune convenzioni epistolari anche all'interno dell'epigramma, molti spunti sono rilevanti anche per il nostro assunto).

19. Impeccabile e istruttiva la definizione che Nisbet - Hubbard 1978, 253, danno dell'otium all'interno dell'ode 2,16 di Orazio: «to literary men like Horace it [scil.: otium] had the more positive qualities of Greek $\sigma \times 0 \lambda \eta$, and suggested the peace and quiet necessary for creative activity» (opportuni poi i confronti con Hor. Epist. 1,7,35-36, Verg. Georg. 4,563 e Ov. Trist. 1,1,41). In età augustea, il tema dell'otium può (non necessariamente deve...) trovare il suo ambito di applicazione all'interno della recusatio: sulla questione, la bibliografia è sterminata e mi limito qui a rimandare a Nauta 2006, 21-27, che affronta il problema in rapporto alla recusatio d'età flavia.

20. Su questo concetto di otium letterario in Marziale, basti qui il rinvio a Gómez Pallarès 1995, nonché alle considerazioni in Rimell 2008, 9, 17 e passim.

21. Sull'importanza dell'immagine di Mecenate nella poesia non solo marzialiana, ma più in generale dei primi due secoli dell'Impero, cfr. Bellandi 1995.

22. Otium e desidia, inertia, segnities e simili spesso sono usati contestualmente, fin dalle prime fasi della letteratura latina, ad indicare una deplorevole indolenza e pigrizia, se non inerzia da debosciati: cfr. Plaut. Asin. 253 dormitasti in otio; quin tu abs te socordiam omnem reice et segnitiem amove; Merc. 62; Cic. Inv. 1,22 eorum inertia, neglegentia, ignavia, desidiosum studium et luxuriosum otium e il materiale raccolto in ThlL IX 2,1166,29-65 e 1179,48-73, s. v. (E. Baer). Questa idea marzialiana dell'otium, invece, come condizione ideale per una alacre attività di composizione di poesia elevata è tutt'altro che scontata nel periodo storico in cui egli vive, se consideriamo che «Stazio, Valerio e Silio sviluppano sempre più nella loro epica spazi più o meno controllati in cui la letteratura dell'otium e del godimento si offre come alternativa, ritardo e sabotaggio del progetto epico» (Barchiesi 2001, 354).

23. Nauta 2006, 38, osserva come il nesso pingue solum richiami, rovesciandolo maliziosamente, l'ideale callimacheo della tenuitas. Direi anche che il lettore, la cui memoria poetica è stata già sollecitata al v. 3 in direzione del celebre incipit delle Bucoliche, coglie nell'allegoria finale ai vv. 7-8 un chiaro, ancorché 'straniante' richiamo all'ideale del labor georgico virgiliano, cfr. Citroni 1975, 328.

24. In ciò, come notava sempre Citroni 1975, ad loc. (326-327), c'è il rovesciamento del motivo tradizionale, secondo il quale l'autore adduce a scusa per non essersi impegnato in un genere elevato l'insufficiente ispirazione e capacità poetica. In 1,107 emerge un'idea dell'otium che Gómez Pallarès 1995, 68-77 definisce come litteratum proprium, in cui la poesia è «protagonista activa del otium». Cfr. poi Howell 1980, 328; soprattutto Nauta 2006, 37-38; e ora Roman 2014, 318-319.

25. Paradigmatico Tibull. 1,1,57 s.: non ego laudari curo, mea Delia; tecum / dum modo sim, quaeso segnis inersque vocer, in cui segnis e iners hanno indubbie connotazioni in senso militare, come ben rilevato nel commento di Maltby 2002, 143.

26. A quel che sembra, Marziale non nega l'accusa di desidia in se stessa: piuttosto, egli afferma che questa pigrizia è forzata, perché inutile è l'impegno in un campus sterile. L'allegoria in cui vengono contrapposti i campi che non danno frutto e il pingue solum, tra l'altro, introduce già in questo I libro il tema della vita beata di campagna sotto una nuova luce: più di una volta essa, 
nell'opera marzialiana, non si coniuga con un ideale di sobrietà 'arcaica', come ad esempio nella letteratura filosofica o satirica, quanto piuttosto di una paradossale 'opulenza' rustica rispetto alla vita grama nella città (il tema percorre tutti i libri di Marziale, e conosce realizzazioni nel segno dell'elogium celebrativo, come in 3,58, oppure della satira, come in 3,47: esso troverà forse la sua più compiuta espressione nel XII libro; cfr. Merli 2006). Il fatto davvero notevole è che in questo passo tale 'ricchezza' campagnola è vista come il frutto del labor, certo sotto la spinta di suggestioni virgiliane (vd. supra, n. 23).

27. Vd. supra, n. precedente.

28. Cfr. Nauta 2006, 38-40; importanti riflessioni sulla poetica espressa in Mart. 8,3 sono in Canobbio 2005, 137-145, e Canobbio 2014, 453-455, con ulteriore bibliografia (si aggiunga Degl'Innocenti Pierini 1999, 173-174, e infine Merli 2013, 128-129).

29. Cfr. lo stesso Citroni 1975, 327, nonché già Izaac 1933, 4, che così traduce i vv. 11-12: «dis-moi, que feras-tu de mieux si tu t'adonnes à la paresse ?»; Pitcher 1998, 64-65; Mattiacci 2007a, 193.

30. Secondo Pitcher 1998, 65 (cfr. poi Mattiacci 2007a, 193), il termine desidiosus farebbe parte di quel linguaggio da eroina elegiaca relicta in cui si esprime la Musa Thalia: di esso si hanno sicure tracce al v. 11 (ingrate, relinquere). Il riferimento sarebbe più esattamente al celebre carme del motto militat omnis amans, Ov. Am. 1,9 (v. 46 qui nolet fieri desidiosus, amet). Insomma, il motivo della desidia elegiaca era già gustosamente capovolto da Ovidio, e Marziale, come lui, parlerebbe di una 'militanza' nel genere 'dolce' e umile delle nugae (non più elegiache, bensì epigrammatiche), rispetto alla quale sarebbe da 'imbelli' sottrarsi ai propri obblighi. Cfr. anche Neger 2012, 289 n. 103, nell'ambito di una discussione sul rapporto tra 1,107 e 8,3 (a 150-156 la studiosa, però, si dedica in modo specifico a 8,3, traducendo in modo 'aperto' a p. 151: «sag mir, was wirst du dann als Müßiger Besseres tun?»

31. Schöffel 2002, 97 e 109-110. Cfr. anche Degl'Innocenti Pierini 1999, 134 n. 106.

32. Ciò ha portato alcuni interpreti a parlare (forse con un po' di esagerazione) di 'fraintendimento' da parte di Talia: cfr. la discussione riguardo a questa linea esegetica in Lorenz 2002, 172-177; Mattiacci 2007a, 193; buone osservazioni in Merli 2013, 128-129 (il motivo del pudor da parte di Marziale nel continuare a scrivere poesia va visto in connessione anche ai temi sviluppati nella sequenza finale del libro VII). Respinge l'idea del 'fraintendimento' anche Neger 2012, 151-152, sostenendo che la Musa risponde all'ego tenendo presente il retroterra degli ipotesti poetici cui Marziale allude in 8,3,1-8, a cominciare (soprattutto) da Ov. Am. 3,1,15-30.

33. In Ovidio stesso la desidia è l'ambito in cui eros attecchisce: cfr. Rem. 149 e 162.

34. È pur vero che in 10,70,2 e in 12 pr. l'accusa di desidia sarà causata dagli indugi da parte di Marziale nella composizione o nella pubblicazione di libri epigrammatici. In questo senso, potrebbe essere letta anche l'accusa della Musa in 8,3,12.

35. Sul significato di questa immagine nella poesia d'età flavia, cfr. ancora Barchiesi 2001, 343-348. Quanto, invece, al tema dei carmi invigilata lucernis, uno dei revisori anonimi mi segnala che, all'interno della tradizione già ellenistica del tema, Callim. Epigr. 27 Pf. = AP 9,507 = G.-P. lvi parlava dell'agrypnia di Arato.

36. Cfr. l'analisi di Lorenz 2002, 177-180.

37. Cfr., dopo Citroni 1968, 288-289, Canobbio 2005, 150-151.

38. Almeno fin da Dau 1887, 76; cfr. soprattutto Fowler 1995, 34.

39. Cfr. Schöffel 2002, 66-67.

40. Mattiacci 2007b, 184-185.

41. Merli 2013, 167-178.

42. Cfr. Merli 2013, 24-41. Si veda in particolare non solo e non tanto il motivo dei labores (che però valgono nel contesto soprattutto come 'affanni, sofferenze') che affievoliscono l'ingenium in Pont. 3,4,11 nos, quibus ingenium longi minuere labores, quanto quello della materia che fa le veci dell' ingenium e dell'ars e diviene ingeniosa in Trist. 5,1,27-28 non haecingenio, non haec componimus arte: / materia est propriis ingeniosa malis (a loro volta, in Ovidio queste formulazioni 
riprendono, per contrasto, altre precedenti: Am. 3,1,25 materia premis ingenium e soprattutto Ars 2,34 materia, qua sis ingeniosus, habes). Se in Ovidio la materia è costituita dai mala che derivano dall'essere caduto in disgrazia presso l'imperatore, in Marziale (in modo parallelo e contrario) essa deriva esattamente dal favore di cui il poeta gode presso Domiziano.

43. Cfr. Mart. 8, praef. 4-5 quamvis autem epigrammata a severissimis quoque et summae fortunae viris ita scripta sint, ut mimicam verborum licentiam adfectasse videantur, ego tamen illis non permisi tam lascive loqui quam solent. Cum pars libri et maior et melior ad maiestatem sacri nominis tui alligata sit, meminerit non nisi religiosa purificatione lustratos accedere ad templa debere; carme 1: laurigeros domini, liber, intrature penates / disce verecundo sanctius ore loqui. / Nuda recede Venus; non est tuus iste libellus: / tu mihi, tu Pallas Caesariana, veni. Cfr. Lorenz 2002, 166-169; Schöffel 2002, 50-86; Neger 2012, 142-146, con ulteriore bibliografia (che si dedica soprattutto al rapporto intertestuale con Ovidio). Giustamente Canobbio 2005, 136, parla di «controcanto rispetto all'epistola I», la prima epistola prefatoria di Marziale in cui l'allegoria voleva che si entrasse nel theatrum del poeta e che si assistesse allo spettacolo licenzioso dei Florales, che prevedeva l'esibizione di mimae nude.

44. Cfr. i relativi commenti di Moreno Soldevila 2006, 183 e 513; Kay 1985, 53-57. In 11,1 è interessante vedere come il motivo assuma una configurazione a tre poli: da una parte c'è il libro, otiosus (v. 1), che ha il tempo di recarsi in giro per trovare lettori, dall'altra il potente e indaffarato patronus Partenio, che non ha la possibilità di vacare, di avere tempo libero per frivolezze come un libro di epigrammi (vv. 4-5); la folla dei romani al portico di Quirino, di cui non esiste nulla di otiosior (v. 10), potrà fornire il pubblico adatto al libellus marzialiano.

45. Cfr. Bowie 1988, 210-211; Craca 2011, 74-77. In generale, sul tema dell'otium nel XII libro di Marziale, cfr. Merli 2006, 341-344; Rimell 2008, soprattutto 190-206; ora Sparagna 2016, in particolare 169-174.

46. In generale sull'importanza del tema dei Saturnali in Marziale, cfr. il contributo di Citroni 1989, in particolare 215. Riguardo all'XI libro, dove il tema è di particolare importanza per la strutturazione del liber, cfr. anche Kay 1985, 5 e 71-72; Merli 1993, 252-253; Scherf 2001, 56-58; sul rapporto tra Nerva e i Saturnalia, cfr. Holzberg 2002, 148-150, e quindi Lorenz 2002, 210-219, e Nauta 2002, 437-440.

47. Esattamente a questo aspetto è dedicato un capitolo del libro di Merli 2013, cfr. in particolare 154-159 (per il tema della festinata cura) e 159-167 (per il ruolo del lettore, patronus, insieme 'correttore' e garante dell'opera); particolare importanza hanno le ripetute riprese del motivo nelle Silvae di Stazio (cfr. in particolare Silv. 1 praef. 30; 1,5,8-14; 4,7,1-12, cui specialmente si dedica Merli 2013, 182-189; a proposito di Silv. 1,5, cfr. ora anche Morelli 2018).

48. Cfr. Coleman 2006, ad loc., 260-262, nonché Lorenz 2002, 67-68.

49. 12 praef: studui paucissimis diebus, ut familiarissimas mihi aures tuas exciperem adventoria sua. $\mathrm{Tu}$ velim ista, quae tantum apud te non periclitantur, diligenter aestimare et excutere.

50. 12,1: retia dum cessant latratoresque Molossi / et non invento silva quiescit apro, / otia, Prisce, brevi poteris donare libello / hora nec aestiva est nec tibi tota perit.

51. Probabilmente ha ragione Sparagna 2014 nel sostenere che il carme 1 doveva riferirsi originariamente ad un libellus diverso offerto a Prisco: il carme poi, insieme ad altri che originariamente facevano parte di altri contesti, finì nella sequenza proemiale del XII libro (cfr. almeno 1-14), creando dei notevoli effetti all'interno del raffinato dispositivo metapoetico che apre l'ultimo libro di Marziale. I problemi connessi all'epistola prefatoria del XII libro, nel suo rapporto con il carme 1, sono affrontati da Craca 2011, 19-46, cui si rimanda anche per la nutrita bibliografia.

52. I lemmi di Xenia e Apophoreta sciorinano già da soli, per il lettore frettoloso, la varietà dei temi marzialiani: essi sono «una sorta di enciclopedia dell'universo elegante e affluente di età flavia» (Moretti 2010, 330; cfr. anche Socas 2004). Per altri versi, la polemica metaletteraria contro il lettore frettoloso, che opera una lettura capricciosa e 'selettiva' dell'opera del poeta, è 
particolarmente frequente, in Marziale, laddove si tratta di giustificare la pratica dell'epigramma longum: mi limito a rimandare a quanto scrivevo in Morelli 2008b, particolarmente 40-43.

53. Cfr. Fusi 2006, ad loc., 209-224, e vd. infra.

54. Cfr. supra, n. 46.

55. Le $\delta \varepsilon ́ \lambda \tau o r$ sono propriamente le 'tavolette', ma per estensione designano in greco qualunque materiale scrittorio (cfr. Floridi 2007, 121); soprattutto, direi, qui si allude in modo divertito a celebri brani di poesia ellenistica, in cui è questione dell'alta ispirazione e del lavoro di fine

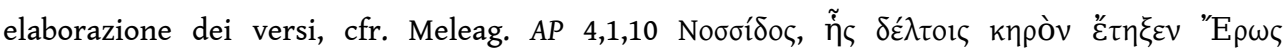
(interessante il confronto con IG IV 1,491,1-2 in Floridi 2007 ibid.).

56. Cfr. Hallett 1996, 336-337; De Miguel Mora 2008, 93.

57. Tabellis sono le tavolette scrittorie: per la questione e per tutti i problemi connessi al rapporto tra esercitazione poetica, poesia e simposio nel carme 50 di Catullo, nonché al rapporto tra c. 50 e successivo c. 51 (con la relativa bibliografia), rinvio a Morelli 2016.

58. Su questi aspetti, cfr. l'analisi di Citroni $2008,44-47$, che valorizza bene la sicura allusione in Priap. 1 non solo a Mart. 1,4,2 (pone supercilium: la medesima espressione anche in Priap. 1,2, vd. supra, § 2) ma anche a 5-6 qua Thymelem spectas derisoremque Latinum / illa fronte precor carmina nostra: Citroni dimostra efficacemente che è il poeta dei Priapea a imitare Marziale, non viceversa, e si tratta di un argomento molto importante anche per la questione relativa alla datazione dell'opera (vd. infra).

59. Höschele 2010, 279.

60. Per la differenza tra poeta epigrammatico e scariphator di versi osceni e aggressivi, è eloquente Mart. 12,61, in particolare i vv. 7-10: quaeras, censeo, si legi laboras, / nigri fornicis ebrium poetam, /qui carbone rudi putrique creta / scribit carmina, quae legunt cacantes.

61. Un gesto per certi versi simile, come mi suggerisce uno dei revisori, è quello iniziale di Persio, nel genere della satira: il poeta si presenta come semipaganus (ipse semipaganus / ad sacra vatum carmen adfero nostrum) dopo essersi sottratto alla consueta convenzione letteraria dell'ispirazione alle fonti delle Muse (vv. 1-6). Andrà notato però che Persio, proprio nella sua qualità di 'semirustico', porta la sua poesia alle sacre cerimonie dei 'cantori ispirati', in un rovesciamento di valori tra 'poesia dello stomaco' e 'poesia laureata' (con burlesca nuova 'eziologia' dell'ispirazione artistica) che viene poi più compiutamente definito ai successivi vv. 8-14. Impossibile qui dar conto anche solo sommariamente dell'ampio dibattito sul significato di semipaganus, per la cui interpretazione seguo ancora comunque, in buona sostanza, Kißel 1990, 86.

62. Sulla retorica apologetica di Priapo nel c. 3, dopo le istruttive considerazioni di Buchheit 1962, 37, interessanti considerazioni sono in Prioux 2008, 163 n. 26 e 171 (vd. anche infra, n. 71). Riflette sulla caratterizzazione 'di genere' del lettore Plantade 2008, 101-102, discutendo delle idee sostenute da Richlin $1992^{2}$, 58 e 121.

63. Su questo celebre epigramma, che cita al suo interno un carme di Augusto imperatore assunto a paradigma della 'semplicità romana' (Romana simplicitas, v. 10), cfr. ora Mattiacci 2016. Sul Latine dicere nei Priapea, cfr. Young 2015.

64. Sull'alternanza metrica come principio costruttivo dell'intero libellus dei Priapea, cfr. Höschele 2008a; Kloss 2003.

65. Priap. 4 stabilisce «une relation entre livret, image et action gestuelle» (Callebat 2008, 31). Lo spazio 'epigrammatico' in cui si collocherà il libro di Priapo viene adeguatamente caratterizzato dal primo dei carmi votivi.

66. Non a caso, si tratta di un altro di quei carmi in cui Priapo, ridotto a rozza statua campestre, riprende e stravolge la lingua tecnica della filologia alessandrina (in particolare, dell'esegesi omerica), con sicuro effetto umoristico: cfr. Höschele 2008b.

67. Sul punto, cfr. Goldberg 1992, 78, e soprattutto Plantade 2008, 108-109; ora Callebat 2012, ad loc. 
68. Nel c. 5 , chiunque entra potrà 'prendere impunemente' (sumas impune) ciò che ritiene opportuno ma dovrà 'dare' (si dederis) ciò che il dio desidera (e che egli già ha esplicitato nel c. 3); nel c. 41 tutti coloro che entreranno nel giardino di Priapo 'offriranno' qualcosa (dei versi) al dio e solo chi non lo farà subirà ciò che viene minacciato nei cc. 3 e 5 . A questo proposito, Vallat 2012, 24, ha parlato di una 'nuova legge' di Priapo nel c. 41, rispetto a quella del c. 5; a me sembra che, nel c. 41 , si prescriva a coloro che entrano nel giardino/tempio del dio un comportamento sulla falsariga di quanto l'ego poetico ha già suggerito nel c. 2 (vd. infra). Io credo che si precisi via via una rete oppositiva tra chi entra in modo 'devoto' nello spazio sacro di Priapo, per donare ed offrire il proprio omaggio (versi, carmi votivi) e chi invece in tale spazio entra per profanarlo o minacciarlo: ladri e poeti docti sono comicamente posti sullo stesso piano.

69. Sul c. 41 e sul suo significato metapoetico, cfr. ancora De Miguel Mora 2008, 86, che rileva una possibile allusione a Catull. 57 (e sia in erudituli di Catull. 57 sia in inter eruditos di Priap. 41, come $\mathrm{mi}$ fa notare uno dei revisori, può benissimo esservi, oltre al primo significato, un double entendre di carattere sessuale, anche se vanno tenute presenti le mie osservazioni in Morelli 2008a, 529), mentre Lhommé 2008 opportunamente studia l'importanza strutturale del tema dell'offerta rituale' nelle sue diverse configurazioni all'interno di Priap. 40-42. In particolare, l'offerta di una corona da parte dell'etera Teletusa nel c. 40 trova eco nell'offerta dei versi da parte dei poeti nel c. 41; parallelamente, all'inizio della raccolta la preghiera iniziale di accettare l'offerta' di versi a Priapo (2,11 in partem accipias bonam, rogamus) trovava corrispondenza poco sotto nella preghiera della prima figura di dedicante, la fanciulla del c. 4 (v. 3 dat donum Lalage rogatque). Cfr. anche Höschele 2010, 291-292, secondo la quale il carme «dürfte ganz einfach als Eröffnungsgedicht für den zweiten Teil des Korpus konzipiert sein».

70. Sulla (anti)poetica di 'degradazione' dell'estetica del libellus nei Carmina Priapea, con particolare riferimento ai carmi proemiali, importanti riflessioni ancora in Höschele 2010, soprattutto 272-282 (e sul valore metapoetico anche di Priap. 14, nel più ampio contesto del libellus, cfr. 288-290).

71. A proposito del carattere di explicit del c. 80, cfr. Höschele 2008a (nonché Höschele 2008b, 384-386, e 2010, 302-304) e Prioux 2008; adesso Morelli 2017, 145. Fin dal c. 3, l'affermazione crassa Minerva mea est in realtà esibisce una paradossale doctrina allusiva e alessandrina, come ha ben notato Prioux 2008, 163 n. 26 (il riferimento è all'identica iunctura in Hor. Sat. 2,3,2, lì riferita al rusticus Ofellus, sapiente 'deviante', abnormis sapiens, personaggio tutto costruito da Orazio sull'asse di una contrapposizione straniante tra tenue e crassum; alla citazione oraziana al c. 3 fanno da pendant proprio alla fine della raccolta, nel c. 80, le allusioni all'Ars e in generale alla lingua tecnica della critica letteraria in Orazio, ravvisate ancora da Prioux 2008). Priapo è in

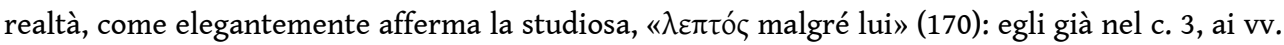
1-8, fa mostra della doctrina retorica (e mitologica: cfr. vv. 5-6, con l'allusione al mito di Ganimede) che nega di possedere. Secondo Vallat 2012, 25-27, il c. 79, che precede immediatamente l'explicit, annuncerebbe la 'fuoriuscita' del dio Priapo (non più prigioniero del suo hortus) dal genere 'minore' dell'epigramma e dalla maschera che egli vi ha assunto per l'intero libellus.

72. Opportunamente Buchheit 2007, è tornato su entrambe le questioni, dopo che Tränkle 1999 aveva messo in dubbio l'unitarietà del libellus e proposto per esso una datazione più alta, e Kloss 2003 aveva espresso cautela; le tesi di Buchheit, d'altro canto, avevano trovato nuovi argomenti a favore grazie all'analisi di Holzberg 2005 (cfr. soprattutto 368-370); cfr. poi Citroni 2008, 49-50; Höschele 2010, 272-273Vallat 2012. Naturalmente, esula dai limiti di questo lavoro un riesame complessivo di tutti gli aspetti connessi ai due problemi: è significativo, comunque, che anche l'indagine che qui si propone vada nella direzione indicata. 


\section{RIASSUNTI}

This paper focuses on the relationship between otium and literature in the Carmina Priapea. In the double prologue of the collection (Priap. 1-2), although lexemes and idioms are similar to those which can be found in most epigrammatic authors, there are significant differences in the use of literary topoi connected to the theme of otium. The new idea of poetry as a 'rough' offering of a supposedly 'idling' poet in Priapus' sacred space takes shape through a number of refined allusions to Catullus, Martial, and also (with high probability) to Strato's Paidike Mousa, in the most important metapoetic poems of the Priapea $(1-2,41,47,49,80)$. This suggests that the collection is the work of a unique author largely inspired by Martial's epigrammatic corpus (Mart. 1,1-4, 1,107, 8 pr., 8,3, 8,55, 11,1-6, 12 pr., and 12,1 are re-examined).

\section{INDICE}

Mots-clés : Carmina Priapea, M. Val. Martialis, Strato Sardensis, otium, liber epigrammaton

\section{AUTORE}

\section{ALFREDO MARIO MORELLI}

Università di Cassino e del Lazio Meridionale 